\title{
Early bladder dysfunction in multiple system atrophy: who seek shall find
}

\author{
Claire Richard $^{1}$. Gérard Amarenco ${ }^{2}$. Jose-Alberto Palma ${ }^{3} \cdot$ Horacio Kaufmann $^{3}$. Sophie Drapier ${ }^{4}$. Xavier Gamé ${ }^{5}$. \\ Benjamin Brucker ${ }^{6} \cdot$ Benoit Peyronnet $^{1}$
}

Received: 4 November 2019 / Accepted: 5 November 2019 / Published online: 8 November 2019

(c) Springer-Verlag GmbH Germany, part of Springer Nature 2019

Multiple system atrophy (MSA) is a fatally progressive neurodegenerative disorder characterized by variable combinations of parkinsonism, cerebellar ataxia and autonomic dysfunction [1]. Even though MSA and Parkinson disease (PD) share some features, MSA is more aggressive and more often characterized by detrusor underactivity, sphincter denervation, and incomplete bladder emptying alone or in association with storage symptoms [2]. Mounting evidence shows that genitourinary symptoms typically appear earlier than motor dysfunction, and early erectile dysfunction or urinary incontinence often precede a diagnosis of MSA [3].

In this issue of Clinical Autonomic Research Sakakibara and colleagues [4] enlighten us about how often and how early bladder autonomic dysfunction predates motor dysfunction in MSA. In this prospective cohort study, the authors found that $18.2 \%$ of MSA patients presented with isolated urinary symptoms, in the absence of movement problems. Because severe urinary symptoms occur early, patients suffering from MSA are very likely to be seen by urologists first, particularly if the patient has no or subtle motor abnormalities [5]. Not infrequently, patients with MSA are misdiagnosed as having benign prostate

Claire Richard

clairerichard3@orange.fr

1 Department of Urology, University of Rennes, Rennes, France

2 Sorbonne Université, GRC 01 GREEN, APHP, Hôpital Tenon, Paris, France

3 Department of Neurology, New York University, New York, USA

4 Department of Neurology, University of Rennes, Rennes, France

5 Department of Urology, University of Toulouse, Toulouse, France

6 Department of Urology, New York University, New York, USA hyperplasia and undergo transurethral resection of the prostate (TURP). This has very poor outcomes, resulting [6, 7] in worsened incontinence and persistent incomplete bladder emptying in most cases which stresses the importance for the urologists of identifying these patients.

By underscoring that urinary symptoms may predate other autonomic, motor or cerebellar symptoms in $\sim 20 \%$ of patients with MSA, the study by Sakakibara and colleagues raises the question of which urinary markers should raise suspicion of an underlying neurological disorder. The authors suggest some possible urological "red flags" in that regard: (1) a large post-void residual urine volume $>100 \mathrm{ml}$ without prostatic hyperplasia in men, (2) an open bladder neck during the filling phase on videourodynamics, and (3) sphincter denervation on electromyography (EMG). We agree with these suggestions, but only within a staged algorithm: incomplete bladder emptying with no obvious cause (e.g. larger prostate, pelvic organ prolapse, diabetes) requires videourodynamics. In case of an open bladder neck, diagnostic confirmation through EMG might be required. However, the diagnostic values of those two tests is unclear. EMG requires a rigorous protocol, which was not well described in the study by Sakakibara and colleagues. The "neurogenic alterations" found in patients with MSA (e.g. abnormalities of recruitment pattern, positive sharp waves, spontaneous fibrillations, pudendal stretch neuropathy) are poorly specific in these age group as they can be result from other causes (e.g. lumbar spinal stenosis or childbirth). To support their results, urodynamics shall include a formal pressure flow study, especially in men, which was not detailed here. Moreover, owing to their costs and invasiveness, EMG and urodynamics cannot be considered screening tools. Future prospective studies should define precise, non-invasive urological markers predicting the development of MSA, as it has been performed with orthostatic hypotension [8].

Early recognition of MSA is key for the urologists to tailor management of urinary symptoms and to avoid harmful 
surgical procedures. However, whether early diagnosis of urinary dysfunction impacts survival of MSA is yet undefined. Whereas MSA remains a fatally progressive neurodegenerative disease [9], urosepsis is a common cause of death, so it might be tempting to hypothesize that early diagnosis and proper management of urinary dysfunction in MSA may have a positive impact on survival. In any case, adequate diagnosis and management of urinary dysfunction in MSA is key to avoid unnecessary surgery and improve selection of pharmacological therapy, thus reducing the burden of side effects.

There is no question that urologists need a call to attention to screen for MSA more efficiently. The study by Sakakibara and colleagues should raise awareness among urologists on the fact that patients with MSA frequently present with isolated urinary dysfunction because, in the very own words of Sophocles, only those who seek shall find.

Funding None.

\section{Compliance with ethical standards}

Conflict of interest The author(s) declare that they have no conflict of interest.

\section{References}

1. Palma JA, Norcliffe-Kaufmann L, Kaufmann H (2018) Diagnosis of multiple system atrophy. Auton Neurosci 211:15-25
2. Peyronnet B, Manunta A, Game X (2015) Multiple-system atrophy. N Engl J Med 372(14):1374-1375

3. Fowler CJ (2007) Update on the neurology of Parkinson's disease. Neurourol Urodyn 26(1):103-109

4. Sakakibara R, Panicker J, Simeoni S, Uchiyama T, Yamamoto T, Tateno F et al (2018) Bladder dysfunction as the initial presentation of multiple system atrophy: a prospective cohort study. Clin Auton Res. https://doi.org/10.1007/s10286-018-0550-y (in press)

5. Beck RO, Betts CD, Fowler CJ (1994) Genitourinary dysfunction in multiple system atrophy: clinical features and treatments in 62 cases. J Urol 151(5):1336-1341

6. Sakakibara R, Panicker J, Finazzi-Agro E, Iacovelli V, Bruschini $\mathrm{H}$, The Parkinson's Disease Subcomittee, The Neurourology Promotion Committee in The International Continence Society (2016) A guideline for the management of bladder dysfunction in Parkinson's disease and other gait disorders: a guideline for Bladder Dysfunction in PD \& Other Gait disorders. Neurourol Urodyn 35(5):551-563

7. Chandiramani VA, Palace J, Fowler CJ (1997) How to recognize patients with parkinsonism who should not have urological surgery. Br J Urol 80:100-104

8. Kaufmann H, Norcliffe-Kaufmann L, Palma JA, Biaggioni I, Low PA, Singer W, Goldstein DS, Peltier AC, Shibao CA, Gibbons $\mathrm{CH}$, Freeman R, Robertson D, Autonomic Disorders Consortium (2017) Natural history of pure autonomic failure: a United States prospective cohort. Ann Neurol. 81(2):287-297

9. Wenning GK, Scherfler C, Granata R, Bosch S, Verny M, Chaudhuri KR et al (1999) Time course of symptomatic orthostatic hypotension and urinary incontinence in patients with postmortem confirmed parkinsonian syndromes: a clinicopathological study. J Neurol Neurosurg Psychiatry 67(5):620-623 